\title{
Le Port de Québec comme mégaprojet : Une insertion territoriale difficile
}

\author{
Paul Villeneuve ${ }^{1}$
}

\section{INTRODUCTION}

\section{Depuis quelques temps, le Port de Québec fait la manchette pour les mauvaises raisons : poussière rouge de nickel, émanant d'activités de transbordement.}

Depuis quelques temps, le Port de Québec fait la manchette pour les mauvaises raisons : poussière rouge de nickel, émanant d'activités de transbordement, qui se répand dans Limoilou; silos d'entreposage de granules de bois inflammables installés à l'Anse au Foulon à quelques mètres du prolongement de la Promenade Samuel de Champlain, un équipement récréotouristique majeur; refus de se soumettre aux règlements du ministère de l'Environnement du Québec ${ }^{2}$.

Ce n'est pas la première fois que les activités industrialo-portuaires provoquent la critique dans la région de Québec. Pourtant, le Port est une institution vénérable dont l'histoire est indissociable de celle de la ville. Pourquoi, dès lors, cette association devient-elle problématique aujourd'hui? Le poids des activités portuaires dans l'économie régionale a diminué au cours des ans, mais les visées de l'Administration portuaire de Québec (APQ), considérées dans leur ensemble, n'en constituent pas moins un mégaprojet territorial capable de modifier en profondeur le paysage de Québec. Comment faire en sorte que ces visées s'insèrent correctement dans l'évolution de la ville et de sa région? Au moment où Québec se prépare à amplifier son rôle de point d'ancrage du développement nordique, il devient crucial de rassembler les conditions qui permettront à son port de contribuer pleinement à ce rôle.

Les lignes qui suivent évoquent d'abord très brièvement la relation historique entre Québec et son port. Elles s'attachent ensuite à tenter de comprendre pourquoi cette relation est aujourd'hui tumultueuse. Elles explorent enfin des pistes qui, peut-être, pourraient permettre une meilleure insertion territoriale de ce mégaprojet que constitue le Port de Québec.

\section{HIER : LE FLEUVE ET LE PORT EN SYMBIOSE AVEC LA VILLE}

\section{Le Port de Québec est le plus vieux port au Canada. Il est le $2^{\mathrm{e}}$ en importance au Québec et le dernier port du Saint-Laurent en eau profonde avant les Grands Lacs.}

Le Port de Québec est le plus vieux port au Canada. Il est le $2^{\mathrm{e}}$ en importance au Québec et le dernier port du Saint-Laurent en eau profonde avant les Grands Lacs. À l'époque de la voile, la construction navale à Québec constituait une activité intensive en main-d'œuvre et à forte valeur ajoutée. La construction navale et les activités portuaires jouaient alors un rôle de première importance dans l'économie régionale et le commerce international. Vers 1860 à Québec, au moins 30000 travailleurs et leurs familles, sur une population totale de 60000 , dépendaient des activités portuaires liées au commerce du bois. Cette primauté prit fin avec le passage de la voile à la vapeur, les navires à vapeur pouvant remonter le fleuve jusqu'à Montréal beaucoup plus facilement que les voiliers. Ce changement technologique, conjugué à d'autres facteurs, provoqua un ralentissement dans la croissance économique et démographique de Québec ${ }^{3}$.

Une reconversion économique suivit, à compter de la décennie de 1860, dans laquelle le port ne tint pas une place aussi centrale qu'à l'époque de la voile, exception faite de l'aménagement du Bassin Louise inauguré en 1890. Cette reconversion était due essentiellement à une main- 
d'œuvre abondante travaillant dans des fabriques, surtout de cuir et de chaussures, pour les marchés de Montréal et des provinces de l'Atlantique. Dès lors, le port et le fleuve deviennent pour la population des quartiers ouvriers de Québec un lieu de récréation, comme l'atteste la plage de l'Anse au Foulon. En 1885, le grand escalier du marché Finlay donnait un accès grand public au fleuve là où se trouve aujourd'hui la Place de Paris. Les fermiers des alentours, venant vendre leurs produits au marché, y accostaient leurs embarcations. Graduellement, tout au long du $20^{\mathrm{e}}$ siècle, l'accès de la population et des visiteurs au fleuve devient un enjeu, d'autant plus que Québec connaît alors, sur les berges du fleuve, une industrialisation limitée, mais passablement lourde.

De cette époque subsiste un grand chantier naval, La Davie, localisée à Lévis en face de Québec. Subsistent aussi de l'époque industrielle, une papetière, la White Birch, localisée à l'embouchure de la rivière Saint-Charles et, de création beaucoup plus récente, une raffinerie, l'Ultramar, sise sur la rive sud en face de Québec. Ces trois sites industriels lourds se trouvent maintenant à l'intérieur du périmètre urbain de Québec, à l'instar de l'ensemble des propriétés administrées par l'APQ qui totalisent $35 \mathrm{~km}$ carrés de plan d'eau et plus de 200 hectares de terrains aménagés situés dans quatre secteurs portuaires soient l'Anse au Foulon, la Pointe à Carcy, l'Estuaire et Beauport. Cependant, depuis quelques décennies, c'est la transition vers le tertiaire qui constitue l'évolution la plus marquante de la société et de l'économie de Québec.

Graduellement, tout au long du $20^{\mathrm{e}}$ siècle, l'accès de la population et des visiteurs au fleuve devient un enjeu, d'autant plus que Québec connaît alors, sur les berges du fleuve, une industrialisation limitée, mais passablement lourde.

\section{AUJOURD'HUI : DIVERGENCES DANS LA RELATION VILLE-PORT}

Comment le port s'insère-t-il, aujourd'hui, dans cette transition vers le tertiaire? D'une part, il y participe pleinement en développant des activités de croisière internationale; d'autre part, il contribue à freiner cette transition en continuant à développer le transbordement de vrac en milieu urbain.

À l'instar de plusieurs ports dans le monde, le Port de Québec comporte un «Vieux Port », la Pointe à Carcy. Les quais de la Pointe à Carcy ont été rénovés au cours des années 1980. Il s'agit d'un lieu très populaire auprès des résidents de Québec et des visiteurs, notamment les croisiéristes. De la Pointe à Carcy, les vues sur la ville et sur le fleuve sont assez remarquables. L'arrivée à quai est tout particulièrement saisissante. L'industrie des croisières met en valeur la beauté du site de Québec. Jusqu'ici, la cohabitation des résidents de Québec et des croisiéristes à la Pointe à Carcy se déroule bien. Si ces derniers continuent à augmenter en nombre comme ils l'ont fait au cours des années récentes, cette cohabitation pourrait devenir plus difficile. Afin d'accommoder plusieurs navires en même temps, le port songe à utiliser les quais de
l'Anse au Foulon et le quai Paquet localisé à Lévis en face de Québec. Mais le port continue à transborder des matières en vrac à l'Anse au Foulon. La cohabitation entre ce type d'activité et l'accueil de navires de croisières risque de devenir inconfortable.

Le Port de Québec est spécialisé dans le transbordement de vrac solide et liquide, les marchandises générales étant plutôt réservées au port de Montréal. Cette spécialisation provient du fait que le Canada est encore un producteur important de matières premières, notamment le bois, les céréales et les minerais. Le cantonnement du Port de Québec dans le vrac viendrait en partie de la concurrence de Montréal comme terminal de conteneurs. En 1978, le Canadien Pacifique ferme son terminal à Québec et concentre à Montréal ses activités de manutention de conteneurs ${ }^{4}$. Les seuls conteneurs que l'on retrouve sur le territoire du Port de Québec contiennent des marchandises générales destinées au ravitaillement de mines situées dans le Grand Nord canadien, un marché qui prend de l'expansion. 
Le transbordement de vrac est une activité à faible valeur ajoutée. Le poids des activités de transbordement de vrac dans l'économie régionale est faible. Il est de l'ordre de $0,5 \%$ à $1 \%$ du produit intérieur brut de la Région métropolitaine de Québec.

Le transbordement de vrac est une activité à faible valeur ajoutée. Le poids des activités de transbordement de vrac dans l'économie régionale est faible. Il est de l'ordre de $0,5 \%$ à $1 \%$ du produit intérieur brut de la Région métropolitaine de Québec et serait assez stable dans le temps, alors que la contribution au PIB de la RMR de Québec d'une activité typiquement tertiaire, comme les services professionnels, scientifiques et techniques, a augmenté de 3,5\% à 5\% entre 1997 et 2009. Les personnes travaillant dans le tertiaire moteur sont généralement fortement scolarisées et se préoccupent de leur qualité de vie, assez pour mettre sur pied, dans le cas qui nous occupe, une «Initiative citoyenne de vigilance du Port de Québec » si elles constatent que l'intégrité de leur milieu de vie est menacée. Une proportion non négligeable de ces travailleurs et travailleuses contribue à la gentrification des quartiers voisins du port. Les motivations de ces personnes peuvent être d'ordre écologique. Elles visent aussi à préserver leur investissement dans l'achat d'une maison dans ces quartiers. Nous ne disposons pas à ce jour d'études comparatives de l'impact social et économique de la gentrification et du transbordement de vrac. Il y a fort à parier que les activités portuaires qui nuisent à la revalorisation des quartiers centraux de Québec ne représentent pas un gros bénéfice net pour la collectivité ${ }^{5}$.

\section{DEMAIN : NOUVELLE GOUVERNANCE ET CHANGEMENT D'ÉCHELLE}

\section{L'APQ est consciente des difficultés associées au transbordement de vrac à proximité de quartiers résidentiels dans une ville qui se pique d'offrir à ses citoyens un milieu urbain d'excellente qualité.}

L'APQ est consciente des difficultés associées au transbordement de vrac à proximité de quartiers résidentiels dans une ville qui se pique d'offrir à ses citoyens un milieu urbain d'excellente qualité. Deux types de démarches sont envisagés pour tenter de surmonter ces difficultés. D'une part, l'APQ mise sur de meilleures relations avec le public, en développant de nouvelles formes de gouvernance ${ }^{6}$. D'autre part, en élargissant l'échelle de sa gestion territoriale, elle explore la possibilité de collaborer avec d'autres ports du Saint-Laurent dont les infrastructures sont sous-utilisées.

Comment l'APQ s'y prend-elle pour améliorer son image? Dans son rapport annuel pour l'année 2012, le président-directeur général exprime ses regrets en ce qui concerne l'émission de poussière dans un quartier voisin :

«Si éclatante soit-elle, l'année 2012 fut assombrie par un incident d'émission de poussière à l'extérieur des limites géographiques du Port de Québec. En effet, un quartier voisin des installations portuaires a été incommodé par de la poussière provenant d'une activité portuaire. Il m'est impossible de passer ce regrettable incident sous silence à l'intérieur de ma rétrospective annuelle. ${ }^{7}$

Le texte ne précise pas qu'il s'agit de poussière de nickel et que, selon la Direction régionale de la santé publique, le seuil acceptable de nickel dans l'air de Limoilou est dépassé depuis $15 \mathrm{ans}^{8}$. L'APQ met alors sur pied un comité de relations avec la communauté :

«Il est primordial de créer des liens durables avec la communauté. C'est précisément en vue de réaliser cet objectif que fut créé le comité de relations avec la communauté lors de la réunion publique annuelle du 31 mai 2012. Ce comité est composé de 15 membres représentant tous les groupes d'intérêts gravitant autour du Port de Québec.»"

La Ville de Québec et les citoyens concernés mettent également sur pied deux autres comités pour surveiller les problèmes de pollution en provenance du port. Le comité de vigilance par rapport aux activités portuaires a comme mandat d'examiner les impacts environnementaux et sanitaires des activités portuaires à Québec. Le comité est consultatif et jouit d'un pouvoir de 
recommandation. Il se compose de citoyens et de représentants des gouvernements provincial et municipal ainsi que du Port de Québec. Le comité intersectoriel sur la contamination environnementale dans l'arrondissement de La CitéLimoilou a été mis sur pied par la Direction régionale de santé publique et vise la réduction à la source de la pollution et la mise en place de mesures correctives ${ }^{10}$. Cette multiplication des comités de surveillance et de vigilance fait craindre les dédoublements et les omissions, mais le fait que les pouvoirs publics et les citoyens aient leur mot à dire est de bon augure aux yeux de plusieurs.

Cependant, le comportement un peu cavalier du Port dans le récent dossier des silos d'entreposage de granules de bois à l'Anse au Foulon montre que la multiplication des comités peut ne pas avoir beaucoup d'impact sur le terrain ou, du moins, ne pas avoir produit à ce jour des résultats concrets. La nouvelle gouvernance se veut collaborative et territorialisée. Elle tente de prendre en compte les intérêts des acteurs qui cohabitent un territoire. Ceci n'est pas toujours facile. Des blocages d'ordre constitutionnel doivent également être surmontés. Tant que le Port se repliera sur son statut d'institution fédérale pour se soustraire, lorsqu'opportun, aux normes provinciales et municipales, toute forme de gouvernance large aura du mal à s'exercer correctement. Cette situation entraîne en effet des problèmes de gouvernance. Par exemple, l'APQ se dit soumise à la seule compétence d'Ottawa alors qu'Environnement Canada soutient que les problèmes de qualité de l'air sont de compétence provinciale ${ }^{11}$.

Par ailleurs, et de façon légitime, l'APQ poursuit une vigoureuse politique de croissance des activités de transbordement de vrac liquide et

\section{CONCLUSION}

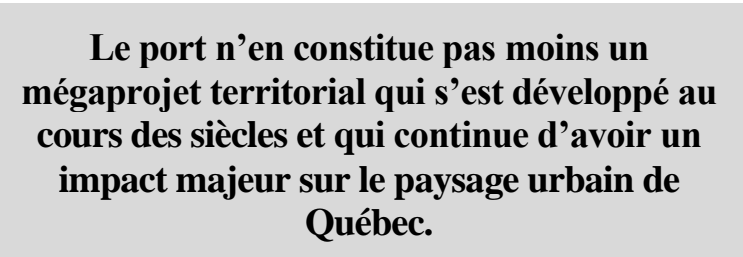

Le Port de Québec contribue de moins en moins au produit intérieur brut de la région de Québec, solide. Tant que cette croissance sera concentrée sur les terrains actuels du Port, au sein du milieu urbain, les trois comités mis sur pied auront sans doute de nombreux cas de pollution à discuter. Une solution à plus long terme n'est-elle pas de sortir le vrac des milieux urbains, d'autant plus que le port manque d'espace comme l'APQ est la première à le reconnaître? En limitant le transbordement du vrac aux berges étroites du fleuve au sein de la capitale, l'Administration portuaire de Québec freine le développement d'une gestion multisectorielle des rives du fleuve à l'échelle de l'ensemble du territoire québécois. Les infrastructures portuaires hors des grands centres urbains doivent être considérées. C'est tout récemment ce qui semble être le cas. Comme en Colombie Britannique, où le Port de Prince Rupert accueille le trop plein du port de Vancouver, les ports du Saint-Laurent en aval de Québec, au premier chef Gros-Cacouna, pourraient désengorger le Port de Québec. Le débat d'il y a quelques années autour des projets d'implantation de terminaux méthanier à Gros-Cacouna et à Lévis (projet Rabaska) montre l'intérêt de considérer les milieux moins densément peuplés pour y implanter certains types d'infrastructure lourde ${ }^{12}$.

D'ailleurs, des discussions ont lieu depuis quelques mois entre l'APQ et la Commission régionale du Port de Gros-Cacouna concernant un possible partenariat. Selon le pdg de l'APQ, Gros-Cacouna pourrait éventuellement accueillir le trop plein de navires du Port de Québec, pourvu que les cargos et les produits s'y prêtent. En échange, le Port de Québec pourrait faire connaître Gros-Cacouna en le présentant à des clients potentiels au niveau international. Dans l'axe du Saint-Laurent, les ports ont intérêt à collaborer plutôt qu'à se faire concurrence $^{13}$.

du moins en ce qui concerne le transbordement de vrac liquide et solide. Pourtant, le port n'en constitue pas moins un mégaprojet territorial qui s'est développé au cours des siècles et qui continue d'avoir un impact majeur sur le paysage urbain de Québec. Les installations du port occupent des terrains stratégiques sur les berges du fleuve. Ces installations participent d'une économie extractive : on transborde à Québec des 
produits primaires peu ou pas transformés. Ce type d'activité s'apparente à l'industrie lourde avec son cortège de nuisances. On peut penser que c'est par inertie que le transbordement de vrac continue à se faire en milieu urbain, même s'il s'agit d'un milieu urbain dont la qualité est exceptionnelle. On peut évoquer des raisons environnementales et sociales pour justifier une relocalisation des activités de transbordement de vrac en dehors du périmètre urbain de Québec, mais il y a aussi des raisons purement économiques: la beauté des paysages de Québec et la qualité de la vie dans les quartiers centraux ont un prix. Ce prix est incorporé dans les valeurs immobilières des quartiers centraux. On peut se demander si la valeur ajoutée au PIB régional par le transbordement de vrac est supérieure ou inférieure à la valeur ajoutée par la beauté des paysages et la qualité de la vie.

\section{La beauté des paysages de Québec et la qualité de la vie dans les quartiers centraux ont un prix. Ce prix est incorporé dans les valeurs immobilières des quartiers centraux.}

\section{BIBLIOGRAPHIE ET NOTES}

${ }^{1}$ L'auteur est professeur retraité de l'Université Laval et membre du Centre de recherche en aménagement et développement (CRAD).

${ }^{2}$ MORIN, A. «Silos à l'Anse au Foulon : les risques d'incendie inquiètent plus que l'aspect visuel » $L e$ Soleil, 12 novembre 2013; Annie Morin «Arrimage Québec pressée de se soustraire aux lois sur l'environnement » Le Soleil, 18 décembre 2013.

${ }^{3}$ LEMELIN, A. (1981). «Le déclin du port de Québec et la reconversion économique à la fin du XIX ${ }^{\mathrm{e}}$ siècle. Une évaluation de la pertinence de l'hypothèse du staple », Recherches sociograpiques, vol. $22, \mathrm{n}^{\mathrm{0}} 2$, p. $155-186$.

${ }^{4}$ CHARLIER, J. (1988). «La conteneurisation du trafic des marchandises diverses et le nouveau jeu de la concurrence portuaire dans l'est du Canada » Norois, $\mathrm{n}^{\mathrm{o}} 137$, p. $35-55$.

${ }^{5}$ LACOURCIÈRE, J. (2012). "Impact en vrac : les retombées économiques dans la région de Québec du transport maritime des marchandises au Port de Québec », Initiative citoyenne de vigilance du Port de Québec, http://www.vigilanceportdequebec.com/wpcontent/uploads/2013/01/Impacts-en-vrac-Mai-2012v2.pdf (consulté le 16 février 2014).
${ }^{6}$ DAUDET, B. et ALIX, Y. (2013). « Gouvernance des territoires ville-port : empreintes locales, concur-rences régionales et enjeux globaux» Organisations et territoires, Vol. 21, $\mathrm{n}^{\mathrm{o}} 3$, p. 41-53.

${ }^{7}$ GIRARD, M., pdg de l'APQ, Rapport annuel 2012, p. 13.

${ }^{8}$ «Poussière de nickel dans Limoilou: les effets sur la santé sont réels», Radio-Canada, 26 avril 2013; http://ici.radio-canada.ca/regions/quebec/2013/04/26/

006-poussiere-nickel-sante-publique-limoilou-

vendredi.shtml (consulté le 22 février 2014).

${ }^{9}$ GIRARD, M., pdg de l'APQ, Rapport annuel 2012, p. 12.

${ }^{10}$ MORIN, A., «Trois comités pour évaluer le Port de Québec », Le Soleil, 8 mai 2013.

${ }^{11}$ MORIN, A. « La poussière rouge balayée dans la cour du provincial », Le Soleil, 19 janvier 2014.

${ }^{12}$ ALIX, Y. et GUY, E. (2007). «Pour une reconsidération des critères d'attractivité territoriale : le cas des projets d'implantation de terminaux portuaires méthaniers au Québec », Organisations et territoires, vol. $16, \mathrm{n}^{\mathrm{o}} 2$ et 3 , p. 115-122.

${ }^{13}$ MORIN, A. «Port de Québec : 2014 sera l'année de la consultation », Le Soleil, 8 janvier 2014. 


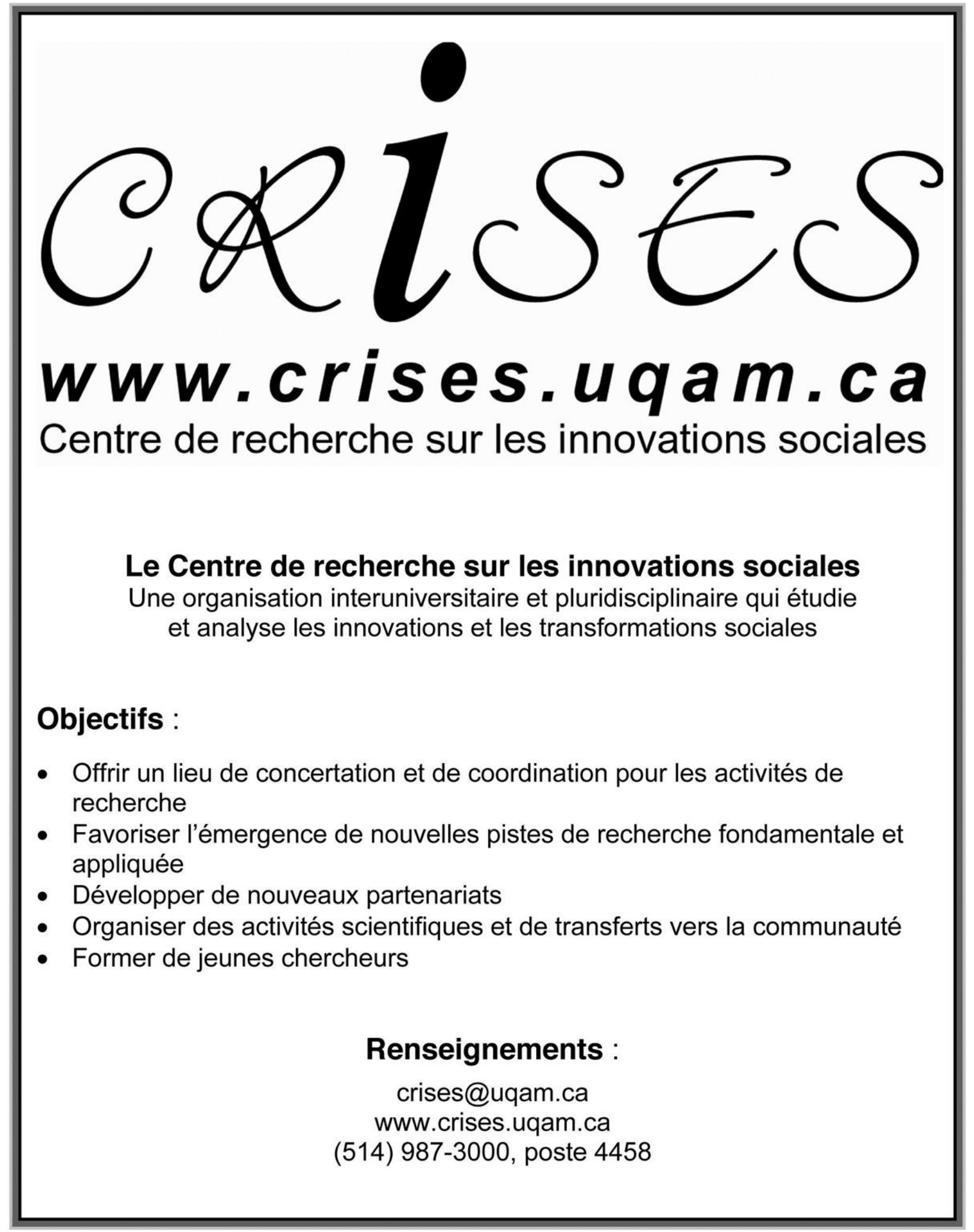

